TRANSACTIONS OF THE

AMERICAN MATHEMATICAL SOCIETY

Volume 355, Number 2, Pages 775-786

S 0002-9947(02)03114-8

Article electronically published on October 1, 2002

\title{
ON THE NONEXISTENCE OF CLOSED TIMELIKE GEODESICS IN FLAT LORENTZ 2-STEP NILMANIFOLDS
}

\author{
MOHAMMED GUEDIRI
}

\begin{abstract}
The main purpose of this paper is to prove that there are no closed timelike geodesics in a (compact or noncompact) flat Lorentz 2-step nilmanifold $N / \Gamma$, where $N$ is a simply connected 2-step nilpotent Lie group with a flat left-invariant Lorentz metric, and $\Gamma$ a discrete subgroup of $N$ acting on $N$ by left translations. For this purpose, we shall first show that if $N$ is a 2-step nilpotent Lie group endowed with a flat left-invariant Lorentz metric $g$, then the restriction of $g$ to the center $Z$ of $N$ is degenerate. We shall then determine all 2-step nilpotent Lie groups that can admit a flat left-invariant Lorentz metric. We show that they are trivial central extensions of the threedimensional Heisenberg Lie group $H_{3}$. If $(N, g)$ is one such group, we prove that no timelike geodesic in $(N, g)$ can be translated by an element of $N$. By the way, we rediscover that the Heisenberg Lie group $H_{2 k+1}$ admits a flat left-invariant Lorentz metric if and only if $k=1$.
\end{abstract}

\section{INTRODUCTION}

Based on an old result due to Tipler [19], it is natural to conjecture that a compact Lorentz manifold admitting a globally hyperbolic regular covering contains a closed timelike geodesic. In a recent work [9] we provided, among other things, examples of compact flat Lorentz space forms without closed timelike geodesics. Since each of such spaces admits Minkowski space as its universal covering, this provides a negative answer to the above conjecture. In this paper we shall show that these examples are not isolated cases, but belong to an infinite family of which they are simplest representatives. Namely, we shall show that given a simply connected 2step nilpotent Lie group $N$ with a flat left-invariant Lorentz metric $g$, then for any lattice $\Gamma$ in $N$, the compact flat Lorentz 2-step nilmanifold $(N / \Gamma, g)$ contains no closed timelike geodesic. This will be a particular case of Theorem 3 . For this, we shall first show in Lemma 3 that the restriction of $g$ to the center $Z$ of $N$ is necessarily degenerate. We then determine all 2 -step nilpotent Lie groups that can admit a left-invariant Lorentz metric with zero sectional curvature. We show in Theorem 1 that they are trivial central extensions of the three-dimensional Heisenberg group $H_{3}$. This, in particular, rediscovers in passing that the generalized Heisenberg Lie group $H_{2 k+1}$ admits a flat left-invariant Lorentz metric if and only if $k=1$. Finally, using the explicit solutions of the geodesic equations in the case where the center is degenerate and some criteria that characterize translated geodesics, both given

Received by the editors July 6, 2001 and, in revised form, June 5, 2002.

2000 Mathematics Subject Classification. Primary 53C22, 53C50; Secondary 53B30.

Key words and phrases. 2-step nilpotent Lie groups, left-invariant Lorentz metrics, closed timelike geodesics. 
in [10, we prove in Theorem 2 that there is no timelike geodesic in $(N, g)$ that can be translated by a nonidentity element of $N$. This implies that, for any discrete subgroup $\Gamma \subset N$ acting on $N$ by left translations (in particular, for any lattice $\Gamma \subset N)$, the flat Lorentz 2-step nilmanifold $(N / \Gamma, g)$ contains no closed timelike geodesic (cf. Theorem 3).

\section{Preliminaries}

Recall that a Lie algebra $\mathcal{N}$ is said to be 2-step nilpotent if it satisfies $[\mathcal{N}, \mathcal{N}] \neq 0$ and $[\mathcal{N},[\mathcal{N}, \mathcal{N}]]=0$. This means that the derived subalgebra $\mathcal{N}^{\prime}=[\mathcal{N}, \mathcal{N}]$ is contained in the center $\mathcal{Z}$ of $\mathcal{N}$, or equivalently, $a d_{X}^{2}=0$ for any $X \in \mathcal{N}$. A Lie group $N$ is said to be 2-step nilpotent if its Lie algebra $\mathcal{N}$ is 2-step nilpotent. Throughout this paper, $N$ will denote a simply connected 2-step nilpotent Lie group with Lie algebra $\mathcal{N}$ having $\mathcal{Z}$ as its center. We shall use $\exp : \mathcal{N} \rightarrow N$ to denote the Lie group exponential map which is, given that $N$ is simply connected and nilpotent, a diffeomorphism (cf. [18, p. 6), and we shall denote its inverse by $\log : N \rightarrow \mathcal{N}$. Since $\mathcal{N}$ is 2-step nilpotent, the Campell-Baker-Hausdorff formula (see for instance [12] or [17]) becomes

$$
\exp (X) \exp (Y)=\exp \left(X+Y+\frac{1}{2}[X, Y]\right), \quad \text { for all } X, Y \in \mathcal{N} .
$$

The differential map of exp is described by the following lemma, which is due to Eberlein [5].

Lemma 1. For any $\xi, A \in \mathcal{N}$, the differential map $D_{\xi} \exp : T_{\xi} \mathcal{N} \rightarrow T_{\exp (\xi)} N$ satisfies

$$
D_{\xi} \exp \left(A_{\xi}\right)=D L_{\exp (\xi)}\left(A+\frac{1}{2}[A, \xi]\right)
$$

where $A_{\xi}$ denotes the initial velocity of the curve $t \mapsto \xi+t A$ and $L_{\exp (\xi)}$ denotes the left translation by $\exp (\xi)$.

As a standard example of a 2-step nilpotent Lie group, we have the following:

Example 1. The Heisenberg group of dimension $2 n+1$.

We call the Heisenberg group of dimension $2 n+1$ the vector space $H_{2 n+1}=$ $\mathbb{R} \times \mathbb{C}^{n}$ endowed with the group law

$$
(z, v) \cdot\left(z^{\prime}, v^{\prime}\right)=\left(z+z^{\prime}+\frac{1}{2} B\left(v, v^{\prime}\right), v+v^{\prime}\right),
$$

where $B$ is the nondegenerate alternate $\mathbb{R}$-bilinear form

$$
B\left(v, v^{\prime}\right)=\sum_{i=1}^{n} x_{i} y_{i}^{\prime}-y_{i} x_{i}^{\prime},
$$

with $v=\left(x_{i}+\sqrt{-1} y_{i}\right)_{1 \leq i \leq n}, v^{\prime}=\left(x_{i}^{\prime}+\sqrt{-1} y_{i}^{\prime}\right)_{1 \leq i \leq n}$ and $z, z^{\prime} \in \mathbb{R}$.

Its Lie algebra $\mathcal{H}_{2 n+1}$ has a basis $\left\{Z, X_{1}, \ldots, X_{n}, \bar{Y}_{1}, \ldots, Y_{n}\right\}$ for which all brackets are zero except $\left[X_{i}, Y_{i}\right]=Z$ for $1 \leq i \leq n$. This means that the center $\mathcal{Z}=\mathbb{R} Z$ of $\mathcal{H}_{2 n+1}$ is 1-dimensional, and it is just straightforward to verify that exp : $\mathcal{H}_{2 n+1}$ $\rightarrow H_{2 n+1}$ is given by

$$
\exp \left(z Z+\sum_{i=1}^{n}\left(x_{i} X_{i}+y_{i} Y_{i}\right)\right)=\left(z+\frac{1}{2} \sum_{i=1}^{n} x_{i} y_{i},\left(x_{i}+\sqrt{-1} y_{i}\right)_{1 \leq i \leq n}\right) .
$$


2.1. Left-invariant Lorentz metrics. Throughout this paper, we shall endow $\mathcal{N}$ $\cong T_{e} N$ with a nondegenerate (indefinite) inner product $\langle$,$\rangle , which in turn induces a$ left-invariant pseudo-Riemannian metric on the Lie group $N$, and we shall restrict to the Lorentz case, that is, we shall assume that $\langle$,$\rangle has signature (-,+, \ldots,+)$. We shall also use the notation $\langle$,$\rangle for both the inner product on \mathcal{N}$ and the corresponding left-invariant Lorentz metric on $N$.

A tangent vector $X$ is said to be timelike, spacelike or lightlike (null) according to whether $\langle X, X\rangle\langle 0,>0$ or $=0$, respectively. A smooth curve in $N$ is said to be timelike, spacelike or lightlike (null) if its tangent vector field is always timelike, spacelike or lightlike (null), respectively. Similarly, a subspace $V \subset \mathcal{N}$ is said to be timelike, spacelike or lightlike (null or degenerate) if the restriction of $\langle$,$\rangle to V$ is indefinite, positive definite or degenerate, respectively.

Definition 1. A 2-step nilpotent Lie group $N$ with a left-invariant Lorentz metric $\langle$,$\rangle will be called a Lorentz 2-step nilpotent Lie group, and will be denoted by$ $(N,\langle\rangle$,$) .$

If the restriction of $\langle$,$\rangle to the center \mathcal{Z}$ is nondegenerate (positive definite or indefinite), let $\mathcal{V}$ denote the orthogonal complement of $\mathcal{Z}$ in $\mathcal{N}$ relative to $\langle$,$\rangle , and$ so write $\mathcal{N}$ as an orthogonal direct sum

$$
\mathcal{N}=\mathcal{V} \oplus Z .
$$

For each $Z \in \mathcal{Z}$, define a skew-symmetric linear map $j(Z): \mathcal{V} \rightarrow \mathcal{V}$ by

$$
j(Z) X=a d_{X}^{*} Z \text { for all } X \in \mathcal{V},
$$

where $a d_{X}^{*} Z$ denotes the adjoint of $a d_{X}$ relative to $\langle$,$\rangle . This equivalently means$ that

$$
\langle j(Z) X, Y\rangle=\langle[X, Y], Z\rangle \text { for all } X, Y \in \mathcal{V} .
$$

Notice that this map was first introduced by Kaplan [13, [14], [15] to study Riemannian 2-step nilmanifolds of Heisenberg type.

Similarly, if the restriction of $\langle$,$\rangle to \mathcal{Z}$ is degenerate, namely if, considered as a vector subspace of $\mathcal{N}$, the center $\mathcal{Z}$ is tangent to the light cone at the identity $e$ of $N$, or equivalently, $\mathcal{Z} \subset b^{\perp}$ for some lightlike vector $b \in \mathcal{Z}$, then one can write $\mathcal{Z}=\mathcal{Z}_{1} \oplus \mathbb{R} b$, where $\mathcal{Z}_{1}$ is a spacelike subspace of $\mathcal{N}$. Since the orthogonal complement $\mathcal{Z}_{1}^{\perp}$ of $\mathcal{Z}_{1}$ in $\mathcal{N}$ is timelike, one can choose a second lightlike vector $c \in \mathcal{Z}_{1}^{\perp}$ to get finally the orthogonal decomposition

$$
\mathcal{N}=Z_{1} \oplus \mathcal{U}_{1} \oplus \operatorname{Vect}\{b, c\}
$$

where $\mathcal{U}_{1}$ is a timelike subspace of $\mathcal{N}$.

For each $z_{1}+y c \in \mathcal{Z}_{1} \oplus \mathbb{R} c$, we might in a similar manner as above define a linear skew-symmetric map $j\left(z_{1}+y c\right) \in \operatorname{End}\left(\mathcal{U}_{1} \oplus V e c t\{b, c\}\right)$ by

$$
j\left(z_{1}+y c\right) X=a d_{X}^{*}\left(z_{1}+y c\right) \text { for all } X \in \mathcal{U}_{1} \oplus V e c t\{b, c\},
$$

or equivalently,

$$
\left\langle j\left(z_{1}+y c\right) X, Y\right\rangle=\left\langle[X, Y], z_{1}+y c\right\rangle \text { for all } X, Y \in \mathcal{U}_{1} \oplus V e c t\{b, c\} .
$$

However, we will need to alter this definition because, as we can easily check, $j\left(z_{1}+y c\right) b=0, \quad j\left(z_{1}+y c\right) c \in \mathcal{U}_{1}$ and $j\left(z_{1}+y c\right) X_{1} \in \mathcal{U}_{1} \oplus \mathbb{R} b$ for all $X_{1} \in \mathcal{U}_{1}$. Instead, we will adopt the following definition:

$$
j: \mathcal{Z}_{1} \oplus \mathbb{R} c \longrightarrow \operatorname{Hom}\left(\mathcal{U}_{1} \oplus \mathbb{R} c, \mathcal{U}_{1} \oplus \mathbb{R} b\right),
$$


where here $\operatorname{Hom}\left(\mathcal{U}_{1} \oplus \mathbb{R} c, \mathcal{U}_{1} \oplus \mathbb{R} b\right)$ denotes the set of all homomorphisms (i.e., linear maps) from $\mathcal{U}_{1} \oplus \mathbb{R} c$ into $\mathcal{U}_{1} \oplus \mathbb{R} b$.

Now, for any $z_{1}+y c \in \mathcal{Z}_{1} \oplus \mathbb{R} c$, we define the linear maps

$$
\begin{aligned}
& J_{1}: \mathcal{U}_{1} \oplus \mathbb{R} c \longrightarrow \mathcal{U}_{1}: X \longmapsto\left(j\left(z_{1}+y c\right) X\right)^{\mathcal{U}_{1}}, \\
& J_{2}: \mathcal{U}_{1} \oplus \mathbb{R} c \longrightarrow \mathbb{R} b: X \longmapsto\left(j\left(z_{1}+y c\right) X\right)^{b},
\end{aligned}
$$

where $\left(j\left(z_{1}+y c\right) X\right)^{\mathcal{U}_{1}}$ and $\left(j\left(z_{1}+y c\right) X\right)^{b}$ are the components of $j\left(z_{1}+y c\right) X$ in $\mathcal{U}_{1}$ and $\mathbb{R} b$, respectively.

One can also define the skew-symmetric endomorphism

$$
J: \mathcal{U}_{1} \longrightarrow \mathcal{U}_{1} \text { by } J(X)=J_{1}(X) \text { for all } X \in \mathcal{U}_{1},
$$

and if $\mathcal{V}_{2}$ denotes the orthogonal complement of $\mathcal{V}_{1}=\operatorname{ker} J$ in $\mathcal{U}_{1}$, then we get the orthogonal decomposition

$$
\mathcal{U}_{1}=\mathcal{V}_{1} \oplus \mathcal{V}_{2}
$$

Since $\mathcal{U}_{1}$ is spacelike and $J$ is skew-symmetric, it follows that the nonzero characteristic roots of $J$ are pure imaginary and occur in conjugate pairs. Therefore, if $\left\{ \pm i \theta_{1}, \ldots, \pm i \theta_{k}\right\}$ are the distinct nonzero characteristic roots of $J$, with $\theta_{i}>0$ for $1 \leq i \leq k$, and $\left\{W_{1}, \ldots, W_{k}\right\}$ are the invariant subspaces of $J$, we have $\mathcal{V}_{2}=\bigoplus_{i=1}^{k} W_{i}$ (orthogonal direct sum) and, relative to an orthonormal basis of $W_{i}$, we can write

$$
J_{\left.\right|_{W_{i}}}=\left(\begin{array}{cc}
0 & \theta_{i} \\
-\theta_{i} & 0
\end{array}\right) \text {. }
$$

\subsection{Covariant derivative and curvature.}

Definition 2. Let $(N,\langle\rangle$,$) be a Lorentz 2-step nilpotent Lie group with non-$ degenerate center. A two-dimensional subspace $\mathcal{P} \subset T_{x} N, x \in N$, is said to be nondegenerate if the restriction of $\langle,\rangle_{\left.\right|_{\mathcal{P}}}$ is nondegenerate. This means that $\langle X, X\rangle\langle Y, Y\rangle-\langle X, Y\rangle^{2} \neq 0$, for any basis $\{X, Y\}$ of $\mathcal{P}$.

A nondegenerate plane $\mathcal{P}$ is said to be timelike if $\langle,\rangle_{\left.\right|_{\mathcal{P}}}$ is indefinite (that is, with signature $(-,+)$ ). Similarly, we say that $\mathcal{P}$ is spacelike if $\langle,\rangle_{\left.\right|_{\mathcal{P}}}$ is positive definite (that is, with signature $(+,+))$.

If we identify elements of $\mathcal{N}$ with their associated left-invariant vector fields, then for $X, Y \in \mathcal{N}$, the covariant derivative $\nabla_{X} Y$ of the left-invariant Lorentz metric $\langle$,$\rangle satisfies the formula (cf. [3] p. 64, or [12] p. 48)$

$$
\nabla_{X} Y=\frac{1}{2}\left\{[X, Y]-a d_{X}^{*} Y-a d_{Y}^{*} X\right\},
$$

where, as above, $a d_{X}^{*} Y$ and $a d_{Y}^{*} X$ denote respectively the adjoints of $a d_{X}$ and $a d_{Y}$ relative to $\langle$,$\rangle .$

The curvature tensor $R$ is defined by

$$
R(X, Y) W=\nabla_{[X, Y]} W-\nabla_{X} \nabla_{Y} W+\nabla_{Y} \nabla_{X} W
$$

and the sectional curvature of a nondegenerate two-dimensional plane $\mathcal{P}=$ $\operatorname{span}\{X, Y\}$ spanned by two vectors $X, Y \in \mathcal{N}$ is given by

$$
K(X, Y)=\frac{\langle R(X, Y) X, Y\rangle}{\langle X, X\rangle\langle Y, Y\rangle-\langle X, Y\rangle^{2}} .
$$

Remark 1. Rather than considering all sectional curvatures, it suffices for different reasons to restrict to timelike sectional curvatures (see 2], Remark 11.6). 
2.3. Geodesics. The geodesic equations are completely integrable 8 and their solutions are expressed in terms of the initial conditions and the eigenvalues of the maps $j(Z)$ or $j\left(z_{1}+y c\right)$ according to whether $\mathcal{Z}$ is nondegenerate or degenerate, respectively [10. Since we will consider only the case where $\mathcal{Z}$ is degenerate, we state the solutions uniquely for this case.

Let $\gamma(t)$ be a geodesic in $N$ emanating from the identity $e$ of $N$, with $\gamma^{\prime}(0)=$ $z_{10}+y_{10}+z_{0} b+y_{0} c$, where $z_{10} \in \mathcal{Z}_{1}, y_{10} \in \mathcal{U}_{1}$ and $z_{0}, y_{0} \in \mathbb{R}$. Using exponential coordinates, one can write

$$
\gamma(t)=\exp \left(z_{1}(t)+y_{1}(t)+z(t) b+y(t) c\right),
$$

where

$$
z_{1}(t) \in \mathcal{Z}_{1}, \quad y_{1}(t) \in \mathcal{U}_{1} \text { and } z(t), y(t) \in \mathbb{R} \text { for all } t \in \mathbb{R} .
$$

Setting $X(t)=y_{1}(t)+y(t) c$ and using Lemma 1 , one obtains

$$
\begin{aligned}
\gamma^{\prime}(t) & =\left(D_{\log (\gamma(t))} \exp \right)\left(z_{1}^{\prime}(t)+y_{1}^{\prime}(t)+z^{\prime}(t) b+y^{\prime}(t) c\right) \\
& =L_{\gamma(t)^{*}}\left(z_{1}^{\prime}(t)+y_{1}^{\prime}(t)+z^{\prime}(t) b+y^{\prime}(t) c+\frac{1}{2}\left[X^{\prime}(t), X(t)\right]\right),
\end{aligned}
$$

and therefore

$$
L_{\gamma(t) *}^{-1} \gamma^{\prime}(t)=z_{1}^{\prime}(t)+y_{1}^{\prime}(t)+z^{\prime}(t) b+y^{\prime}(t) c+\frac{1}{2}\left[X^{\prime}(t), X(t)\right] .
$$

Note that $z_{1}^{\prime}(0)=z_{10}, y_{1}^{\prime}(0)=y_{10}, z^{\prime}(0)=z_{0}$ and $y^{\prime}(0)=y_{0}$.

Lemma 2 (8]). Let $G$ be a Lie group with a left-invariant pseudo-Riemannian metric induced by a nondegenerate inner product $\langle$,$\rangle on the Lie algebra \mathcal{G}$ of $G$. For any $C^{1}$-curve $t \mapsto \gamma(t)$ in $G$, associate the curve $L_{\gamma(t) *}^{-1} \gamma^{\prime}(t)$ in $\mathcal{G}$. Then, the curves of $\mathcal{G}$ associated to geodesics of $G$ are solutions of the equation

$$
X^{\prime}=a d_{X}^{*} X,
$$

where as above $a d_{X}^{*}$ stands for the adjoint of $a d_{X}$ relative to $\langle$,$\rangle .$

Substituting (4) into (5), we obtain the system

$$
\begin{aligned}
z_{1}^{\prime}(t)+\frac{1}{2}\left[X^{\prime}(t), X(t)\right]^{\mathcal{Z}_{1}} & =z_{10}, \\
\frac{d}{d t}\left(z^{\prime}(t) b+\frac{1}{2}\left[X^{\prime}(t), x(t)\right]^{b}\right) & =J_{2}\left(X^{\prime}(t)\right), \\
X^{\prime \prime}(t) & =J_{1}\left(X^{\prime}(t)\right) .
\end{aligned}
$$

The solution of this system is described by the following result.

Proposition 1 (10]). Using the notation above, let $(N,\langle\rangle$,$) be a simply connected$ Lorentz 2-step nilpotent Lie group with degenerate center. Let $\gamma(t)$ be a geodesic in $N$ emanating from the identity e of $N$ such that $\gamma^{\prime}(0)=z_{10}+z_{0} b+y_{10}+y_{0} c$, where $z_{10} \in \mathcal{Z}_{1}, y_{10} \in \mathcal{U}_{1}, z_{0}, y_{0} \in \mathbb{R}$, and set $\gamma(t)=\exp \left(z_{1}(t)+z(t) b+y_{1}(t)+y(t) c\right)$, where $z_{1}(t) \in \mathcal{Z}_{1}, y_{1}(t) \in \mathcal{U}_{1}$ and $z(t), y(t) \in \mathbb{R}$ for all $t \in \mathbb{R}$. Set also $J_{1}(c)=c_{1}+c_{2} \in \mathcal{V}_{1} \oplus \mathcal{V}_{2}, y_{10}=y_{11}+y_{12} \in \mathcal{V}_{1} \oplus \mathcal{V}_{2}$, and $X_{1}=y_{11}-y_{0} J^{-1} c_{2}$, $X_{2}=y_{12}+y_{0} J^{-1} c_{2}=\sum_{i=1}^{k} \xi_{i}$, where $\xi_{i} \in W_{i}$ for $1 \leq i \leq k$. Then,

(1) $y(t) c=t y_{0} c$;

(2) $y_{1}(t)=t X_{1}+\frac{t^{2}}{2} y_{0} c_{1}+\left(e^{t J}-I d\right) J^{-1} X_{2}$;

(3) $z_{1}(t)=t\left(z_{10}+T_{1}(t)^{\mathcal{Z}_{1}}\right)+T_{2}(t)^{\mathcal{Z}_{1}}$; 


$$
\text { (4) } \begin{aligned}
z(t) b=t\left(z_{0} b\right. & \left.+T_{1}(t)^{b}-J_{2}\left(J^{-1} X_{2}\right)\right)+T_{2}(t)^{b} \\
& +\frac{t^{2}}{2} J_{2}\left(X_{1}\right)+\frac{t^{3}}{6} y_{0} J_{2}\left(c_{1}\right)+J_{2}\left(\left(e^{t J}-I d\right) J^{-2} X_{2}\right),
\end{aligned}
$$

where $T_{1}(t)^{\mathcal{Z}_{1}}, T_{2}(t)^{\mathcal{Z}_{1}}$ (resp. $\left.T_{1}(t)^{b}, T_{2}(t)^{b}\right)$ are the components on $\mathcal{Z}_{1}$ (resp. $\mathbb{R} b)$ of the following quantities:

$$
\begin{aligned}
T_{1}(t)= & \frac{1}{2}\left[X_{1}+y_{0} c+\frac{t}{2} y_{0} c_{1},\left(e^{t J}+I d\right) J^{-1} X_{2}\right] \\
& +\frac{t^{2}}{12} y_{0}\left[X_{1}+y_{0} c, c_{1}\right]-y_{0}\left[c_{1}, e^{t J} J^{-2} X_{2}\right] \\
& -\frac{1}{2} \sum_{i=1}^{k}\left[\xi_{i}, J^{-1} \xi_{i}\right], \\
T_{2}(t)= & y_{0}\left[c_{1},\left(e^{t J}-I d\right) J^{-3} X_{2}\right] \\
& -\left[X_{1}+y_{0} c,\left(e^{t J}-I d\right) J^{-2} X_{2}\right]+\frac{1}{2}\left[e^{t J} J^{-1} X_{2}, J^{-1} X_{2}\right] \\
& -\frac{1}{2} \sum_{i \neq j=1}^{k} \frac{1}{\left(\theta_{i}^{2}-\theta_{j}^{2}\right)}\left(\left[e^{t J} \xi_{i}, e^{t J} \xi_{j}\right]-\left[e^{t J} J \xi_{i}, e^{t J} J^{-1} \xi_{j}\right]\right) \\
& +\frac{1}{2} \sum_{i \neq j=1}^{k} \frac{1}{\left(\theta_{i}^{2}-\theta_{j}^{2}\right)}\left(\left[\xi_{i}, \xi_{j}\right]-\left[J \xi_{i}, J^{-1} \xi_{j}\right]\right),
\end{aligned}
$$

respectively.

\subsection{Translated geodesics.}

Definition 3. Let $\gamma(t)$ be a geodesic in $(N,\langle\rangle$,$) and \phi$ a nontrivial element of $N$. We say that $\phi$ translates $\gamma$ by an amount $\omega>0$ if $\phi \cdot \gamma(t)=\gamma(t+\omega)$ for all $t \in \mathbb{R}$. If $\gamma(t)$ is a unit speed geodesic, $\omega$ is called a period of $\phi$.

When the restriction of $\langle$,$\rangle to \mathcal{Z}$ is degenerate, translated geodesics are characterized by the following result.

Proposition 2 ([10]). Assume that $\mathcal{Z}$ is degenerate and let $\phi$ be an arbitrary element of $N$. Write $\phi=\exp \left(z_{10}^{*}+z_{0}^{*} b+y_{10}^{*}+y_{0}^{*} c\right)$, with $z_{10}^{*} \in \mathcal{Z}_{1}, y_{10}^{*} \in \mathcal{U}_{1}$ and $z_{0}^{*}, y_{0}^{*} \in \mathbb{R}$, and let $\gamma(t)$ be a unit speed or null geodesic such that $\gamma(0)=a=$ $\exp \eta, \gamma(\omega)=\phi . a$ and $\gamma^{\prime}(0)=D L_{a}\left(z_{10}+z_{0} b+y_{10}+y_{0} c\right)$ for suitable $z_{10} \in$ $\mathcal{Z}_{1}, y_{10} \in \mathcal{U}_{1}$ and $z_{0}, y_{0} \in \mathbb{R}$. Set $z_{10}^{* *}+\lambda^{*} b=z_{10}^{*}+z_{0} b+\left[y_{10}^{*}+y_{0}^{*} c, \eta\right]$ and write $\gamma(t)=a \cdot \exp \left(z_{1}(t)+z(t) b+y_{1}(t)+y(t) c\right)$ with $z_{1}(t) \in \mathcal{Z}_{1}, y_{1}(t) \in \mathcal{U}_{1}$ and $z(t), y(t) \in \mathbb{R}$ for all $t \in \mathbb{R}$, and $z_{1}(0)=y_{1}(0)=0, z(0)=y(0)=0$. Then, with the notation above, the following assertions are equivalent:

(1) $\phi . \gamma(t)=\gamma(t+\omega)$ for all $t \in \mathbb{R}$;

(2)

$$
\begin{aligned}
y(t+\omega) c= & \left(y(t)+y_{0}^{*}\right) c \\
y_{1}(t+\omega)= & y_{1}(t)+y_{10}^{*}, \\
z_{1}(t+\omega)+z(t+\omega) b= & z_{1}(t)+z(t) b+z_{10}^{* *}+\lambda^{*} b \\
& +\left[y_{10}^{*}+y_{0}^{*} c, y_{1}(t)+y(t) c\right] \text { for all } t \in \mathbb{R} ;
\end{aligned}
$$

(3) $e^{\omega J} X_{2}=X_{2}, y_{0} c_{1}=0$ and $J_{2}\left(X_{1}\right)=0$. In particular, $\gamma(t)$ cannot be timelike if $c_{1} \neq 0$; 
(4) $y_{0}^{*} c=\omega y_{0} c, y_{10}^{*}=\omega X_{1}$,

$$
\begin{aligned}
z_{10}^{* *}+\lambda^{*} b=\omega & \left\{z_{10}+z_{0} b+\left[y_{0} c+X_{1}, J^{-1} X_{2}\right]+\frac{1}{2} \sum_{i=1}^{k}\left[J^{-1} \xi_{i}, \xi_{i}\right]\right\} \\
& -\omega J_{2}\left(J^{-1}\left(X_{2}\right)\right), \text { where } X_{2}=\sum_{i=1}^{k} \xi_{i} ;
\end{aligned}
$$

(5) $\gamma^{\prime}(0) \perp \mathcal{Z}_{V^{*}} . a$, where $\mathcal{Z}_{V^{*}}=\exp \left(\left[y_{10}^{*}+y_{0}^{*} c, \mathcal{N}\right]\right)$;

(6) $\gamma^{\prime}(\omega) \perp \mathcal{Z}_{V^{*} . \phi . a}=\mathcal{Z}_{V^{*} \cdot \gamma}(\omega)$.

\section{MAIN RESULTS}

We start with the following lemma.

Lemma 3. Let $(N,\langle\rangle$,$) be a flat Lorentz 2-step nilpotent Lie group. Then, the$ restriction of $\langle$,$\rangle to the center \mathcal{Z}$ of $\mathcal{N}$ is degenerate.

Proof. Assume to the contrary that $\langle$,$\rangle is nondegenerate on \mathcal{Z}$, and write as usual $\mathcal{N}=V \oplus Z$, where $\mathcal{V}$ denotes the orthogonal complement of $\mathcal{Z}$ relative to $\langle$,$\rangle . Using$ (11), we see for $x, y \in \mathcal{V}, z \in \mathcal{Z}$ that

$$
\nabla_{x} x=\nabla_{z} z=0, \quad \nabla_{x} y=\frac{1}{2}[x, y], \quad \nabla_{x} z=-\frac{1}{2} j(z) x .
$$

Therefore,

$$
\begin{aligned}
R(z, x) z & =-\nabla_{z} \nabla_{x} z \\
& =\frac{1}{2} \nabla_{z} j(z) x \\
& =-\frac{1}{4}\left(a d_{j(z) x}^{*} z\right),
\end{aligned}
$$

and if $z, x$ are orthonormal, we compute using (3),

$$
\begin{aligned}
K(z, x) & =\epsilon\langle R(z, x) z, x\rangle \\
& =-\frac{\epsilon}{4}\left\langle a d_{j(z) x}^{*} z, x\right\rangle \\
& =-\frac{\epsilon}{4}\langle[j(z) x, x], z\rangle \\
& =\frac{\epsilon}{4}\langle[x, j(z) x], z\rangle \\
& =\frac{\epsilon}{4}\langle j(z) x, j(z) x\rangle,
\end{aligned}
$$

where $\epsilon=-1$ or 1 depending upon whether the plane section $\mathcal{P}=\operatorname{span}\{x, z\}$ is timelike or spacelike, respectively.

Case 1: $\mathcal{V}$ is spacelike. In this case, to raise a contradiction it suffices by (7) to show that there exist $z \in \mathcal{Z}$ and $e \in \mathcal{V}$ such that $j(z) e \neq 0$, because this would imply that $K(z, e) \neq 0$, which is a contradiction given that we are assuming that $R$ is identically zero. To do this, assume first that $[\mathcal{N}, \mathcal{N}]$ is totally degenerate and choose a lightlike vector $b \in \mathcal{N}$ so that $[\mathcal{N}, \mathcal{N}]=\mathbb{R} b$. By setting $b=\left[e, e^{\prime}\right]$ and recalling that a timelike vector is never orthogonal to a lightlike vector, we easily get for any arbitrary timelike vector $z \in \mathcal{Z}$ that

$$
0 \neq\langle b, z\rangle=\left\langle\left[e, e^{\prime}\right], z\right\rangle=\left\langle j(z) e, e^{\prime}\right\rangle .
$$

If $[\mathcal{N}, \mathcal{N}]$ is not totally degenerate, then by choosing $z \in[\mathcal{N}, \mathcal{N}]$ so that $\langle z, z\rangle \neq 0$ and writing $z=\left[e, e^{\prime}\right]$, we obtain

$$
0 \neq\langle z, z\rangle=\left\langle\left[e, e^{\prime}\right], z\right\rangle=\left\langle j(z) e, e^{\prime}\right\rangle .
$$


Thus, in either case, we have shown that $j(z) e \neq 0$ for suitable $z \in \mathcal{Z}$ and $e \in \mathcal{V}$, as desired.

Case 2: $\mathcal{V}$ is timelike. Recall that $\operatorname{dim} \mathcal{V} \geq 2$ since otherwise $\mathcal{N}$ cannot be 2step nilpotent, and let $e, e^{\prime} \in \mathcal{V}$ be two orthonormal vectors so that $\left[e, e^{\prime}\right] \neq 0$. In this case, the word " $e, e^{\prime}$ are orthonormal" means that $\left\langle e, e^{\prime}\right\rangle=0$ and either $\langle e, e\rangle=\left\langle e^{\prime}, e^{\prime}\right\rangle=1$ or $\langle e, e\rangle=-\left\langle e^{\prime}, e^{\prime}\right\rangle=1$. Since $\nabla_{\left[e, e^{\prime}\right]} e=\nabla_{e}\left[e, e^{\prime}\right]$ then, using the first two equations of (6), we easily compute

$$
\begin{aligned}
R\left(e, e^{\prime}\right) e & =\nabla_{\left[e, e^{\prime}\right]} e-\nabla_{e} \nabla_{e^{\prime}} e \\
& =\frac{3}{2} \nabla_{e}\left[e, e^{\prime}\right] .
\end{aligned}
$$

But $\mathcal{N}$ is 2-step nilpotent and $\left[e, e^{\prime}\right] \in \mathcal{Z}$. Thus,

$$
\begin{aligned}
R\left(e, e^{\prime}\right) e & =\frac{3}{4}\left\{\left[e,\left[e, e^{\prime}\right]\right]-a d_{e}^{*}\left[e, e^{\prime}\right]-a d_{\left[e, e^{\prime}\right]}^{*} e\right\} \\
& =-\frac{3}{4} a d_{e}^{*}\left[e, e^{\prime}\right]
\end{aligned}
$$

and hence,

$$
\begin{aligned}
K\left(e, e^{\prime}\right) & =-\frac{3 \epsilon}{4}\left\langle a d_{e}^{*}\left[e, e^{\prime}\right], e^{\prime}\right\rangle \\
& =-\frac{3 \epsilon}{4}\left\langle\left[e, e^{\prime}\right],\left[e, e^{\prime}\right]\right\rangle \\
& =-\frac{3 \epsilon}{4}\left|\left[e, e^{\prime}\right]\right|^{2} \neq 0,
\end{aligned}
$$

where as above $\epsilon=-1$ or 1 depending upon whether the plane section $\mathcal{P}=$ $\operatorname{span}\left\{e, e^{\prime}\right\}$ is timelike or spacelike, respectively. This is a contradiction, thereby showing that $\langle$,$\rangle must be degenerate on \mathcal{Z}$. This completes the proof of Lemma 3

Theorem 1. A connected 2-step nilpotent Lie group $N$ admits a flat left-invariant Lorentz metric if and only if $N$ is a trivial central extension of the three-dimensional Heisenberg Lie group $\mathrm{H}_{3}$. Furthermore, the restriction of such a metric to the factor $\mathrm{H}_{3}$ is necessarily indefinite with degenerate center.

Proof. By Lemma 3 the restriction of $\langle$,$\rangle to \mathcal{Z}$ is degenerate. This means, as noticed in Subsection 2.1, that $\mathcal{Z}=\mathcal{Z}_{1} \oplus \mathbb{R} b$ (orthogonal direct sum) for some lightlike vector $b$. Since the orthogonal complement $\mathcal{Z}_{1}^{\perp}$ of $\mathcal{Z}_{1}$ relative to $\langle$,$\rangle is$ timelike, we may choose a second lightlike vector $c \in \mathcal{Z}_{1}^{\perp}$ so that $\langle b, c\rangle=1$. Thus, we may finally get the desired orthogonal decomposition

$$
\mathcal{N}=Z_{1} \oplus V e c t\{b, c\} \oplus \mathcal{U}_{1}
$$

with $\operatorname{dim} \mathcal{U}_{1} \geq 1$, because $\mathcal{N}$ is 2 -step nilpotent.

We claim that $\operatorname{dim} \mathcal{U}_{1}=1$. Indeed, suppose to the contrary that $\operatorname{dim} \mathcal{U}_{1} \geq 2$. Without loss of generality, assume that $\operatorname{dim} \mathcal{U}_{1}=2$, and let $e_{1}, e_{2}$ be an orthonormal basis of $\mathcal{U}_{1}$. Recalling that $[\mathcal{N}, \mathcal{N}] \subset \mathcal{Z}=\mathcal{Z}_{1} \oplus \mathbb{R} b$, we may write

$$
\begin{aligned}
{\left[c, e_{1}\right] } & =\alpha_{1} b+z_{1}, \\
{\left[c, e_{2}\right] } & =\alpha_{2} b+z_{2}, \\
{\left[e_{1}, e_{2}\right] } & =\alpha b+z,
\end{aligned}
$$

where $z, z_{1}, z_{2} \in \mathcal{Z}_{1}$ and $\alpha, \alpha_{1}, \alpha_{2} \in \mathbb{R}$. 
Using formula (11), a direct computation yields all the covariant derivatives. We find for instance

$$
\begin{aligned}
& \nabla_{c} z_{1}=\nabla_{z_{1}} c=-\frac{1}{2}\left(\left|z_{1}\right|^{2} e_{1}+\left\langle z_{1}, z_{2}\right\rangle e_{2}\right), \\
& \nabla_{z} e_{1}=\nabla_{e_{1}} z=\frac{1}{2}\left(\left\langle z_{1}, z\right\rangle b-|z|^{2} e_{2}\right) .
\end{aligned}
$$

From these calculations we obtain, using formula (2), that

$$
\begin{aligned}
R\left(e_{1}, e_{2}\right) e_{1} & =\frac{3}{4}\left(\left\langle z_{1}, z\right\rangle b-|z|^{2} e_{2}\right), \\
R\left(v, e_{1}\right) v & =\frac{1}{2} R\left(c, e_{1}\right) c \\
& =-\frac{3}{8}\left(\left|z_{1}\right|^{2} e_{1}+\left\langle z_{1}, z_{2}\right\rangle e_{2}\right)+\frac{\alpha}{8}\left(z_{2}+\alpha e_{1}\right)-\frac{\alpha_{2}}{4} z, \\
R\left(v, e_{2}\right) v & =-\frac{3}{8}\left(\left\langle z_{1}, z_{2}\right\rangle e_{1}+\left|z_{2}\right|^{2} e_{2}\right)-\frac{\alpha}{8}\left(z_{1}-\alpha e_{2}\right)-\frac{\alpha_{1}}{4} z,
\end{aligned}
$$

where $v=\frac{b+c}{\sqrt{2}}$. (Notice that $v$ is a unit spacelike vector, and that $e_{1}, e_{2}, v$ are orthonormal.)

Since we are assuming that $R$ is identically zero, we deduce that $\alpha=0$ and $z=z_{1}=z_{2}=0$, because $e_{1}, e_{2}, v$ are linearly independent. Therefore, the structure equations of $\mathcal{N}$ reduce to

$$
\begin{aligned}
{\left[c, e_{i}\right] } & =\alpha_{i} b, \\
{\left[e_{i}, e_{j}\right] } & =0,
\end{aligned}
$$

where $\alpha_{i} \in \mathbb{R}$ and $e_{i}, e_{j}$ belong to an orthonormal basis $\left\{e_{1}, \ldots, e_{k}\right\}$ of $\mathcal{U}_{1}$.

Since $\mathcal{N}$ is 2 -step nilpotent and not abelian, there exists some $\alpha_{i} \neq 0$. Without loss of generality, assume that $\alpha_{1} \neq 0$, say $\alpha_{1}=1$ to simplify. Now, for any $i \in\{2, \ldots, k\}$ with $\alpha_{i} \neq 0$, by replacing $e_{i}$ with $e_{i}^{\prime}=e_{i}-\alpha_{i} e_{1}$ which is still an element of $\mathcal{U}_{1}$, we see that $\left[x, e_{i}^{\prime}\right]=0$ for any $x \in \mathcal{N}$, that is, $e_{i}^{\prime} \in \mathcal{Z}$. But this contradicts the fact that $\mathcal{U}_{1} \cap \mathcal{Z}=\{0\}$. It follows that $\alpha_{i}=0$ for any $i \in\{2, \ldots, k\}$, which in turn implies that $e_{i} \in \mathcal{Z}$ for $i \in\{2, \ldots, k\}$. But, this is also a contradiction for the same reason that $\mathcal{U}_{1} \cap \mathcal{Z}=\{0\}$; thereby we deduce that $\operatorname{dim} \mathcal{U}_{1}=1$.

It follows that $\mathcal{N}$ is a trivial central extension of the Heisenberg Lie algebra $\mathcal{H}_{3}=\operatorname{span}\left\{b, c, e_{1}\right\}$ with $\left[c, e_{1}\right]=b$. Furthermore, it is clear that the restriction of the metric to the factor $\mathcal{H}_{3}$ is indefinite with degenerate center $\mathbb{R} b$. This completes the proof of Theorem 1 .

As a direct consequence of Theorem 1, we have the following result already established in [1], Proposition 3.5.2 (see also 4], p. 16).

Corollary 1. The Heisenberg Lie group $H_{2 k+1}$ admits a flat left-invariant Lorentz metric if and only if $k=1$.

Remark 2. In terms of Proposition 3.1.11 of [1, Theorem 1] says that $N$ admits a flat left-invariant Lorentz metric if and only if its Lie algebra $\mathcal{N}$ is a double extension of a Euclidean abelian Lie algebra, which is here $\mathcal{Z}_{1} \oplus \mathcal{U}_{1}$, by a straight line with respect to $u=D=0, \mu=0$ and $b_{0}=\lambda e_{1}$.

We may also say that $\mathcal{N}$ is obtained from $\mathcal{H}_{3}$ endowed with a left-invariant Lorentz metric for which the center of $\mathcal{H}_{3}$ is degenerate (and so such a metric is flat), by a trivial central extension. In other words, starting from $\mathcal{H}_{3} \cong V e c t\{b, c, e\}$ with $[c, e]=b$, endowed with a Lorentz scalar product $\langle$,$\rangle such that \langle b, b\rangle=\langle c, c\rangle=0$ 
and $\langle b, c\rangle=1$, we add some new central elements $z_{1}, \ldots, z_{k}$ to the center $\mathbb{R} b$ and extend $\langle$,$\rangle so that z_{1}, \ldots, z_{k}$ are orthonormal and $\mathcal{N}=H_{3} \oplus \operatorname{span}\left\{z_{1}, \ldots, z_{k}\right\}$ is an orthogonal direct sum.

We now investigate the nonexistence of closed timelike geodesics in flat Lorentz 2-step nilmanifolds, but let us first recall how the examples of [9], mentioned in the introduction, were obtained. Indeed, we start from Theorem 3.5 of [6], saying that the Heisenberg group $H_{3}$ acts affinely and simply transitively in two different ways on $\mathbb{R}^{3}$. One of these actions preserves a Lorentz metric which corresponds to a left-invariant Lorentz metric on $\mathrm{H}_{3}$ with nondegenerate restriction to the center, and so this is not a flat metric. However, the second action preserves a Lorentz metric which corresponds to a left-invariant Lorentz metric on $H_{3}$ with degenerate restriction to the center, and therefore this is a flat metric.

This last action of $H_{3}$ on $\mathbb{R}^{3}$ is, up to conjugacy in $\operatorname{Isom}\left(\mathbb{R}_{1}^{3}\right)=O(2,1) \ltimes \mathbb{R}^{3}$, an affine action of the following group:

$$
G_{b, c}=\left\{\left(\begin{array}{ccc}
1 & c v & b u+c v^{2} \\
0 & 1 & v \\
0 & 0 & 1
\end{array}\right)\left[\begin{array}{c}
s+(b+c) u v / 2+c v^{3} / 6 \\
u+v^{2} / 2 \\
v
\end{array}\right]\right\}
$$

where $b, c$ are real numbers with $b \neq c$. Notice that $G_{b, c}$ is abelian if and only if $b=c$.

In order to simplify computations we set, for example, $b=0$ and $c=-1$. In this case, we easily check that $G_{0,-1} \cong H_{3}$ acts isometrically and simply transitively on a Minkowski space $\left(\mathbb{R}_{1}^{3}, g\right)$ with respect to coordinates $x, y, z$ in which $g=2 d x d z+d y^{2}$.

If $\{X, Y, Z\}$ is a basis of $\mathcal{H}_{3}$ with structure equation $[Y, Z]=X$, then $g$ corresponds to a left-invariant Lorentz metric on $H_{3}$ defined at the identity $e$ by the inner product $\langle$,$\rangle for which the center \mathcal{Z}=\mathbb{R} X$ is degenerate. Precisely, we have that $\langle X, X\rangle=\langle Y, Y\rangle=0$ and $\langle X, Y\rangle=\langle Z, Z\rangle=1$.

Let $\Gamma$ be a lattice in $G_{0,-1} \cong H_{3}$, namely, $\Gamma$ is a cocompact discrete subgroup of $\operatorname{Isom}\left(\mathbb{R}_{1}^{3}\right)$ acting properly discontinuously and freely on $\mathbb{R}_{1}^{3}$. Then, the Lorentz quotient manifold $M^{3}=\mathbb{R}_{1}^{3} / \Gamma$ is a compact flat space form with the Minkowski space $\left(\mathbb{R}_{1}^{3}, g\right)$ as its universal covering. In particular, $M^{3}$ admits the regular globally hyperbolic covering $\pi: \mathbb{R}_{1}^{3} \rightarrow M^{3}=\mathbb{R}_{1}^{3} / \Gamma$.

Lemma 4. $M^{3}$ contains no closed timelike geodesics.

Proof. Note first that geodesics of $M^{3}$ are projections under $\pi$ of geodesics (i.e., straight lines $)$ in $\mathbb{R}_{1}^{3}$. If $c(t)=\left(\alpha t+x_{0}, \beta t+y_{0}, \gamma t+z_{0}\right)$ is a geodesic in $\mathbb{R}_{1}^{3}$ translated an amount $\omega$ by an element $\phi \in \Gamma$, then the condition $\phi \cdot c(t)=c(t+\omega)$ is equivalent to the following system of equations:

$$
\left\{\begin{array}{l}
-v\left(\beta t+y_{0}\right)-\left(v^{2} / 2\right)\left(\gamma t+z_{0}\right)+s-u v / 2-v^{3} / 6=\alpha \omega \\
v\left(\gamma t+z_{0}\right)+u+v^{2} / 2=\beta \omega \\
v=\gamma \omega
\end{array}\right.
$$

where $s, u, v$ are integers.

Since $t$ is arbitrary, we necessarily get $\gamma=0$. This implies that $g(\dot{c}, \dot{c})=2 \alpha \gamma+$ $\beta^{2}=\beta^{2} \geq 0$, and hence $c$ cannot be timelike.

Remark 3. Examples of 4-dimensional compact flat space forms without closed timelike geodesics can also be obtained using the classification given in [7]. Each of 
such spacetimes is, up to an isometry, of the form $H_{4} / \Gamma$, where $H_{4}$ is the unique 4dimensional 2-step nilpotent Lie group endowed with a left-invariant Lorentz metric for which the one-dimensional subspace $\left[\mathcal{H}_{4}, \mathcal{H}_{4}\right]$ is null, and $\Gamma$ is a lattice in $H_{4}$. Here $\mathcal{H}_{4}$ denotes the Lie algebra of $H_{4}$. It has a basis $\{X, Y, Z, W\}$ for which the only nonzero bracket is $[X, Y]=Z$. Notice that $\mathcal{Z}=\operatorname{span}\{Z, W\}$, that is, $\operatorname{dim} \mathcal{Z}=$ 2 .

As we have already noticed, these examples are not isolated cases, but belong to an infinite family of which they are simplest representatives. Namely, we have the following.

Theorem 2. Let $N$ be a connected 2-step nilpotent Lie group with a flat leftinvariant Lorentz metric $g$. Then there is no timelike geodesic in $(N, g)$ that can be translated by a nonidentity element of $N$.

Proof. Let $\langle$,$\rangle denote the Lorentz scalar product on \mathcal{N}$ induced by $g$. By Theorem $1 \mathcal{N}$ may be put (as a vector space) into an orthogonal direct sum

$$
\mathcal{N}=H_{3} \oplus \mathcal{Z}_{1}
$$

where as usual $\mathcal{H}_{3} \cong \operatorname{Vect}\{b, c, e\}$ with $[c, e]=b$, and $[x, z]=0$ for any $x \in \mathcal{N}$ and $z \in \mathcal{Z}_{1}$. Furthermore, the restriction of $\langle$,$\rangle to \mathcal{H}_{3}$ is indefinite with degenerate center $\mathbb{R} b$. So, without loss of generality, assume that $\langle b, b\rangle=\langle c, c\rangle=0$ and $\langle b, c\rangle=\langle e, e\rangle=1$. Let $\gamma(t)$ be a geodesic in $N$ through the identity of $N$ such that $\gamma^{\prime}(0)=z_{10}+z_{0} b+y_{0} c+y_{1} e$, where $z_{10} \in \mathcal{Z}_{1}$ and $z_{0}, y_{0}, y_{1} \in \mathbb{R}$, and set

$$
\gamma(t)=\exp \left(z_{1}(t)+z(t) b+y(t) c+y_{1}(t) e\right),
$$

where

$$
z_{1}(t) \in \mathcal{Z}_{1} \text { and } z(t), y(t), y_{1}(t) \in \mathbb{R} \text { for all } t \in \mathbb{R}
$$

Using the notation of Subsection 2.1 one can write

$$
\begin{aligned}
& j\left(z_{10}+y_{0} c\right) e=\alpha e+\beta b, \\
& j\left(z_{10}+y_{0} c\right) c=\delta e,
\end{aligned}
$$

and since $j\left(z_{10}+y_{0} c\right)$ is skew-symmetric, it follows from this that

$$
\alpha=\beta+\delta=0 \text {. }
$$

But $[c, e]=b$. Thus,

$$
\begin{aligned}
\delta & =\left\langle j\left(z_{10}+y_{0} c\right) c, e\right\rangle \\
& =\left\langle[c, e], z_{10}+y_{0} c\right\rangle \\
& =y_{0}
\end{aligned}
$$

Hence,

$$
\begin{aligned}
j\left(z_{10}+y_{0} c\right) e & =-y_{0} b, \\
j\left(z_{10}+y_{0} c\right) c & =y_{0} e .
\end{aligned}
$$

From this we deduce that $J \equiv 0$ and that $c_{1}=y_{0} e$ and $c_{2}=0$, where, as in the statement of Propostion [1, $j\left(z_{10}+y_{0} c\right) c=J_{1}(c)=c_{1}+c_{2} \in \mathcal{V}_{1} \oplus \mathcal{V}_{2}$.

Now, if $\gamma(t)$ is translated by an element $\phi \in N$, then by Proposition 2, we should have $y_{0} c_{1}=0$, that is, $y_{0}^{2} e=0$. This implies that $y_{0}=0$. Recalling from Proposition 1 that $y(t) c=t y_{0} c$, it follows that $y(t) \equiv 0$, and that consequently $\gamma(t)$ cannot be timelike. This completes the proof of Theorem 2 . 
Our main result concerning closed timelike geodesics in compact (and even noncompact) flat spacetimes follows now as a consequence of Theorem 2

Theorem 3. Let $N$ be a simply connected 2-step nilpotent Lie group with a flat left-invariant Lorentz metric. Then for any discrete subgroup $\Gamma \subset N$, the flat 2-step nilmanifold $N / \Gamma$ does not contain closed timelike geodesics.

Remark 4. Note that it is just as straightforward to see that even if it does not contain translated timelike geodesics, a 2-step nilpotent Lie group with a flat leftinvariant Lorentz metric should, however, contain translated lightlike geodesics. This can easily be checked in light of the proof of Theorem 2 (for a more general result concerning flat compact space forms, see [9], Theorem 3.3).

\section{REFERENCES}

1. A. Aubert, Structures affines et pseudo-métriques invariantes à gauche sur des groupes de Lie, Thesis, Univ. of Montpellier II, 1996.

2. J.K. Beem, P.E. Ehrlich, and K. Easley, Global Lorentzian Geometry, Second Edition, Monographs and Textbooks in Pure and Applied Mathematics, vol. 202, Marcel Dekker, New York, Inc., 1996. MR 97f:53100

3. J. Cheeger and D. Ebin, Comparison Theorems in Riemannian Geometry, North-Holland, Amsterdam, 1975. MR 56:16538

4. L. A. Cordero and P. E. Parker, Pseudo-Riemannian 2-step nilpotent Lie groups, Preprint (1999).

5. P. Eberlein, Geometry of 2-step nilpotent groups with a left invariant metric, Ann. Scient. École Norm. Sup. 27 (1994), 611-660. MR 95m:53059

6. D. Fried and W.M. Goldman, Three-Dimensional Affine Crystallographic Groups, Adv. in Math. 47 (1983), 1-49. MR 84d:20047

7. D. Fried, Flat Spacetimes, J. Differential Geom. 26 (1987), 385-396. MR 89a:53074

8. M. Guediri, Sur la complétude des pseudo-métriques invariantes à gauche sur les groupes de Lie nilpotents, Rend. Sem. Mat. Univ. Politec. Torino 52 (1994), 371-376. MR 96d:53078

9. M. Guediri, On the existence of closed timelike geodesics in compact spacetimes, Math. Z. 239 (2002), 277-291.

10. M. Guediri, Lorentz geometry of 2-step nilpotent Lie groups, (October 24, 2000) Submitted.

11. S.W. Hawking and G.F.R. Ellis, The large scale structure of space-time, Cambridge Monographs on Mathematical Physics, No. 1, Cambridge Univ. Press, Cambridge, 1973. MR 54:12154

12. S. Helgason, Differential geometry, Lie groups, and symmetric spaces, Pure and Applied Mathematics, Vol. 80, Academic Press, New York, 1978. MR 80k;53081

13. A. Kaplan, Fundamental solutions for a class of hypoelliptic PDE generated by composition of quadratic forms, Trans. Amer. Math. Soc. 258 (1980), 147-153. MR 81c:58059

14. A. Kaplan, Riemannian nilmanifolds attached to Clifford modules, Geom. Dedicata 11 (1981), 127-136. MR 82h:22008

15. A. Kaplan, On the geometry of groups of Heisenberg type, Bull. London Math. Soc. 15 (1983), 35-42. MR 84h:53063

16. K. Nomizu, Left-invariant Lorentz metrics on Lie groups, Osaka J. Math. 16 (1979), 143-150. MR 80g:53048

17. J.F. Price, Lie groups and compact groups, London Mathematical Society Lecture Note Series, No. 25, Cambridge Univ. Press, Cambridge, 1977. MR 56:8743

18. M. S. Raghunathan, Discrete subgroups of Lie groups, Ergebnisse der Mathematik und ihrer Grenzgebiete, Band 68, Springer-Verlag, New York, 1972. MR 58:22394a

19. F.T. Tipler, Existence of closed timelike geodesics in Lorentz spaces, Proc. Amer. Math. Soc. 76 (1979), 145-147. MR 80f:83016

Department of Mathematics, College of Sciences, King Saud University, P.O. Box 2455, Riyadh 11451, Saudi Arabia

E-mail address: mguediri@ksu.edu.sa 